\title{
A 4-yr-old presenting with chronic cough and asymmetrical chest
}

\author{
A.V. Sridhar*, M. Tofeig ${ }^{\#}$
}

\section{Case history}

A 4-yr-old female was referred by her general practitioner with history of recurrent cough of 18 months duration. The cough was predominantly nocturnal with no associated wheeze, fever or systemic symptoms and no symptoms of gastrooesophageal reflux. There were no exercise-induced symptoms. Asthma was suspected and she had been treated with inhaled salbutamol with no significant improvement in her symptoms. There was no history of recurrent chest infections, foreign body aspiration or previous hospital admissions.

On examination her weight was on the 3 rd centile and height on the 50th centile. There were no cyanosis, clubbing or ear, nose and throat abnormalities. She had a markedly asymmetrical chest with less prominent right hemithorax compared with the left and mediastinal shift to the right. Breath sounds were diminished on the right side with no wheeze or crepitations. The rest of the cardiovascular examination was normal. There were no other abnormalities on clinical examination.

In view of her chronic cough and failure to thrive she had a mantoux test, which was strongly positive. She had bacille Calmette-Guerin vaccination at birth and there was no recent contact with tuberculosis (TB). The sputum was negative for Mycobacterium tuberculosis. She was given TB chemoprophylaxis

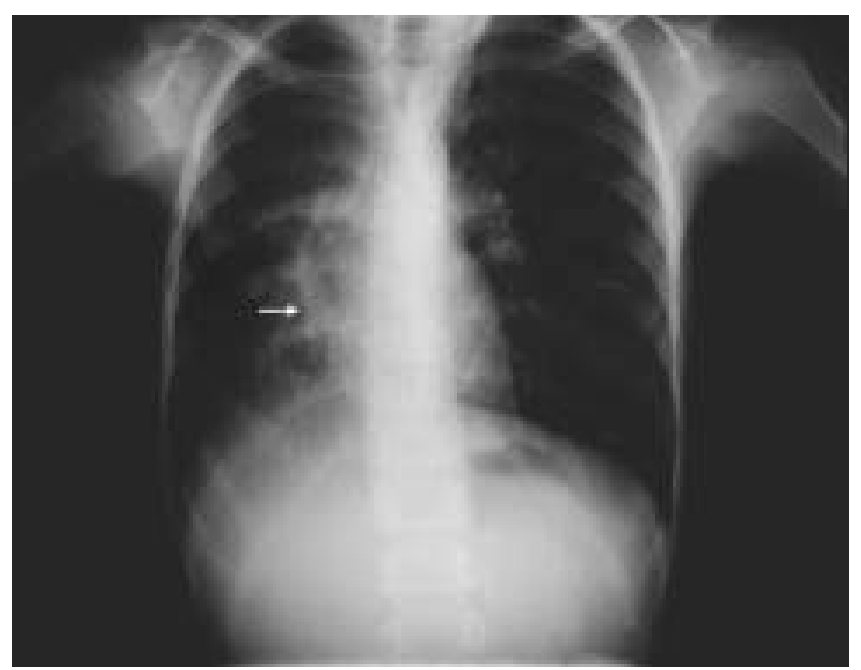

Fig. 1. - Chest radiograph of the patient presenting with asymmetrical chest and chronic cough. with Isoniazid and Rifampicin for 6 months, during which she had a symptomatic improvement in her cough.

A chest radiograph (fig. 1) and computed tomography (CT) scan of the thorax (fig. 2) revealed characteristic findings.

Bronchoscopy revealed abnormalities of the bronchial tree. She had a transthoracic echocardiogram, which revealed characteristic findings. The rest of the cardiac anatomy was normal. She underwent diagnostic cardiac catheterisation, which showed normal right-sided pressures (right atrial mean pressure $4 \mathrm{mmHg}$, right ventricle (RV) pressure 26 over $3 \mathrm{mmHg}$, main pulmonary artery (MPA) 24 over $8 \mathrm{mmHg}$, the right pulmonary artery (RPA) 20 over $8 \mathrm{mmHg}$ and left pulmonary artery (LPA) 20 over $8 \mathrm{mmHg}$ ).

Oximetry showed pulmonary blood flow/systemic blood flow ratio of 1.3:1. The superior vena cava saturation was $83 \%$, right atrial saturation $88 \%$, lower inferior vena cava (IVC) saturation $83 \%$, right atrium (RA)-IVC junction $99 \%$, RV 91\%, MPA 90\%, RPA 90\% and LPA 86\%.

The follow through of the RPA angiogram (fig. 3) revealed the characteristic abnormality. There were no aortic collaterals supplying the lungs. There was no significant intracardiac leftto-right shunt.

Currently she is being managed conservatively with regular clinical and echocardiograph assessment.

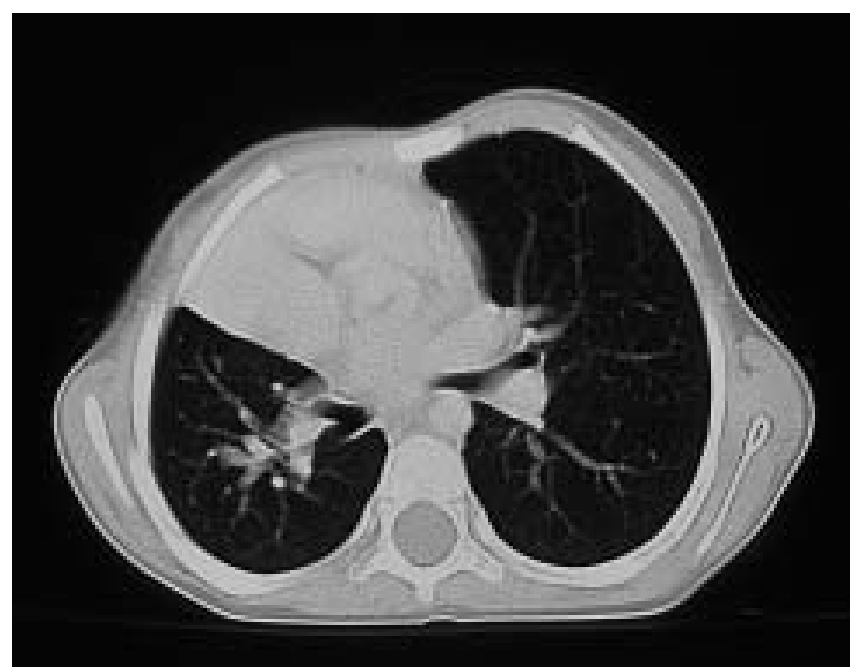

Fig. 2.-Computed tomography of chest $1 \mathrm{~cm}$ below the level of carina.

\footnotetext{
*Dept of Child Health, Leicester University Hospital National Health Service trust, Leicester Royal Infirmary, and ${ }^{\#}$ Dept of Paediatric Cardiology, Leicester University Hospital National Health Service trust, Glenfield Hospital, Leicester, UK.

Correspondence: A.V. Sridhar, Dept of Child Health, Leicester University Hospital NHS trust, Robert Kilpatrick Clinical Sciences Building, Leicester Royal Infirmary, Leicester, LE2 7LX, UK. Fax: 44 1162523282. E-mail: srinaidu@aol.com
} 


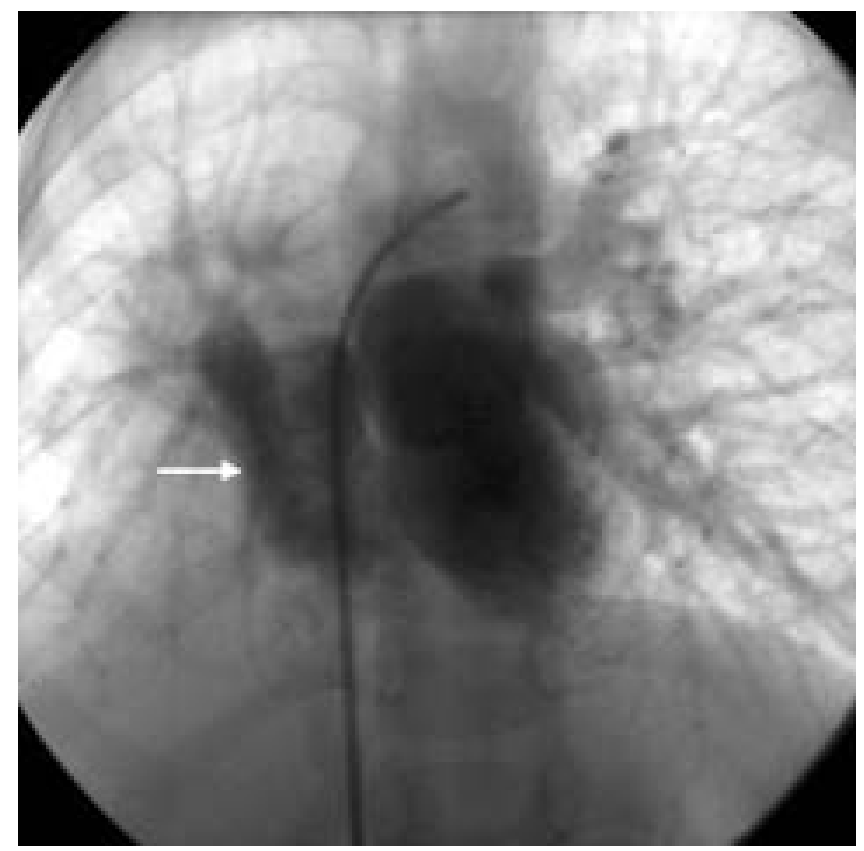

Fig. 3. - Angiogram of the right pulmonary artery.

BEFORE TURNING THE PAGE, INTERPRET THE ROENTGENOGRAM, COMPUTED TOMOGRAPHY SCAN, ANGIOGRAM AND HISTORY AND SUGGEST A DIAGNOSIS. 


\section{Interpretation}

The chest radiograph shows hypoplastic right lung with dextroposition of the heart. The arrow shows a curvilinear density (Scimitar sign) in the right lower zone and 11 pairs of ribs (fig. 1)

The CT of her chest $1 \mathrm{~cm}$ below the level of carina (fig. 2) demonstrates a dextroposition of heart with hypoplastic right lung and absence of right upper-lobe bronchus. Other CT frames showed the absence of right upper lobe.

The RPA angiogram (fig. 3) shows anomalous drainage of the right pulmonary vein (arrow) into the RA and IVC junction.

\section{Diagnosis: "Scimitar syndrome"}

\section{Discussion}

Scimitar syndrome is a rare congenital anomaly consisting of partial anomalous pulmonary venous return from the right lung to the IVC, hypoplasia of the right lung with dextroposition of the heart, and anomalous systemic arterial supply from the abdominal aorta to a portion of right lung (usually lower lobe). Not all patients with scimitar syndrome have all the above abnormalities, but the defining trait is the anomalous pulmonary venous return of all or most of the right lung to the IVC [1].

The anomalous vein travels along the right heart border, curving to the left as it approaches its insertion into the IVC. The radiographic appearance of this anomalous right pulmonary vein resembles a curved Turkish sword or Scimitar, after which this syndrome is named.

Scimitar syndrome has wide spectrum of clinical presentation and can be divided into an infantile form and a paediatric/young adult form [2]. The infantile form generally presents within the first 2 months of life with tachypnoea, failure to thrive and signs of heart failure. The infantile form is a severe form of the disease with major associated cardiac lesions, including atrial-septal defect, ventricular-septal defect, coarctation of aorta, patent ductus arteriosus, and hypoplastic right lung. Pulmonary hypertension is quite common in infants presenting with scimitar syndrome $[1,3]$.

The adult group is defined as those patients in whom the syndrome is detected after the first year of life. In this group the diagnosis is made incidentally and indeed some patients may be completely asymptomatic. Heart failure is usually not present and pulmonary hypertension is uncommon. Some of the common pulmonary anomalies reported with Scimitar syndrome include hypoplastic right lung, pulmonary sequestration, horse-shoe lung and bronchial malformation [4]. The patient in the current case had hypoplastic right lung, along with abnormalities of the right-middle and lower lobe bronchi.

It is interesting to note that in the patient in the current case although the chronic cough was probably related to the diagnosis, the pathophysiology of the symptom remains unclear.

DuPUIS et al. [3], in their series of 122 patients with the adult form of the scimitar syndrome, reported that 38 patients presented with recurrent pneumonia, 23 with exertional dyspnoea, eight with deformation of the thorax, and seven with haemoptysis. NAJIM et al. [4] in their series of 32 patients of whom 13 had the adult form of Scimitar syndrome, reported that two patients presented with fatigue, three with dyspnoea, and four with chest infections. The remaining four were asymptomatic. The other presentations include a 7-yr-old female with the adult form of Scimitar syndrome presenting with recurrent wheeze [5].

In all these cases the relationship between their symptoms and the pathophysiology of scimitar syndrome remains unclear.

The adult form of scimitar syndrome is notoriously difficult to diagnose without a strong index of suspicion because of its varied presentation. In the patient in the current case the asymmetry of the chest on the right side, along with the pattern recognition on the chest radiograph, assisted in the diagnosis.

In many patients clinical examination may be entirely normal. The electrocardiogram may be entirely normal or show right bundle branch block or right ventricular hypertrophy. Echocardiography is extremely useful in diagnosing the scimitar syndrome and its associated cardiac anomalies. Other useful tests in delineating the cardiopulmonary anomalies would include cardiac catheterisation, CT scan and magnetic resonance imaging.

In children with the infantile form of Scimitar syndrome, although repair of the anomalous pulmonary venous return and ligation of collaterals is recommended, right pneumonectomy, either as a primary therapy or if repair fails, have shown similar early and late results [1]. In the adult form of Scimitar syndrome repair is indicated if the shunt is large (pulmonary blood flow/systemic blood flow ratio $>2: 1$ ) [3, 4]. In the patient in the current case pulmonary blood flow/ systemic blood flow ratio was 1.3:1. She is being managed conservatively with regular clinical and echocardiographic assessment. This case highlights the need for a high index of suspicion for this condition in children presenting with asymmetrical chest and chronic cough.

\section{References}

1. Huddleston CB, Exil V, Canter C, Mendeloff EN. Scimitar syndrome presenting in infancy. Ann Thorac Surg 1999; 67: 154-160.

2. Dupuis C, Charaf LAC, Breviere GM, Abou P. The "infantile" form of scimitar syndrome with pulmonary hypertension. Am J Cardiol 1993; 71: 1326-1330.

3. Dupuis C, Charaf LAC, Breviere GM, Abou P, Helmius G. The "adult" form of scimitar syndrome. Am J Cardiol 1992; 70: 502-507.

4. Najim HK, Williams WG, Coles JG, Rebeyka IM, Freedom RM. Scimitar syndrome: twenty years experience and results of repair. J Thorac Cardiovasc Surg 1996; 112: 1161-1168.

5. Desai PR, Babu M. Scimitar syndrome as a differential diagnosis in a child with recurrent wheeze. Arch Dis Child 2002; 87: 357. 